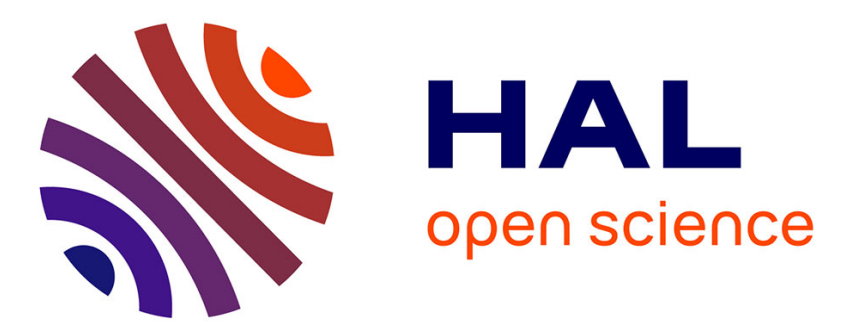

\title{
On the proof of recursive Vogler algorithm for multiple knife-edge diffraction
}

Viet Dung Nguyen, Huy Phan, Ali Mansour, Arnaud Coatanhay, Thierry Marsault

\section{- To cite this version:}

Viet Dung Nguyen, Huy Phan, Ali Mansour, Arnaud Coatanhay, Thierry Marsault. On the proof of recursive Vogler algorithm for multiple knife-edge diffraction. IEEE Transactions on Antennas and Propagation, 2021, 69 (6), pp.3617 - 3622. 10.1109/TAP.2020.3037748 . hal-03104224

HAL Id: hal-03104224

https://hal-ensta-bretagne.archives-ouvertes.fr/hal-03104224

Submitted on 2 Feb 2021

HAL is a multi-disciplinary open access archive for the deposit and dissemination of scientific research documents, whether they are published or not. The documents may come from teaching and research institutions in France or abroad, or from public or private research centers.
L'archive ouverte pluridisciplinaire HAL, est destinée au dépôt et à la diffusion de documents scientifiques de niveau recherche, publiés ou non, émanant des établissements d'enseignement et de recherche français ou étrangers, des laboratoires publics ou privés. 


\title{
Communication On the proof of recursive Vogler algorithm for multiple knife-edge diffraction
}

\author{
Viet-Dung Nguyen, Member, IEEE, Huy Phan, Ali Mansour, Senior Member, IEEE, Arnaud Coatanhay, Thierry \\ Marsault
}

\begin{abstract}
We consider the problem of multiple knife-edge diffraction estimation which is a fundamental task in many wireless communication applications. So far, one of the most accurate methods for this problem is the Vogler one whose recursive implementation is efficient to reduce the high computational complexity of the direct one. However, in the original report, Vogler only presented the final result of the recursive algorithm without a rigorous mathematical proof, thus making the method difficult to understand and implement in practice. To tackle this shortcoming, we first analyze the mathematical structure of the problem and then present a formal proof of the result. To gain intuition of the proof and the key steps, we provide a simplified study case of four knife-edges. The insight from our proposed analysis and proof can be used to obtain a comprehensive interpretation, initiate a practical implementation and develop new efficient algorithms with similar structure.
\end{abstract}

Index Terms-Multiple knife-edge diffraction, Vogler method, recursive algorithm, $\mathbf{R F}$ transmission

\section{INTRODUCTION}

Estimation of diffraction attenuation plays an important role in many applications of wireless communication. Some classical ones can be taken into account such as evaluating propagation loss over irregular terrains [1]-[3] or aeronautical mobile and ground station interactions [4]. More recently, diffraction estimation is also used in several emerged applications, for example, channel modeling at cmWave and mmWave bands for 5G [5] or influence of vehicles as obstacles in Vehicular Ad Hoc Networks (VANET) [6]. Generally, to obtain better accuracy, it is essential to use multiple knifeedge approximation instead of single one which is simple but inadequate.

To date, many approaches are proposed in the literature for calculating the multiple knife-edge diffraction loss. Some well-known representatives includes: (i) 'analytical' methods, such as the Vogler method and the variants of the uniform theory of diffraction (UTD) [7]-[13], to name a few; (ii) graphical-based methods, such as the Bullington method [14], the Deygout method [15], the Causebrook [16] and Giovanelli methods [17]. The first group of methods is characterized

V.-D. Nguyen (corresponding author), A. Mansour and A. Coatanhay are with Lab-STICC, UMR 6285 CNRS ENSTA Bretagne, Brest, France (email: viet.nguyen, ali.mansour, arnaud.coatanhay@ensta-bretagne.fr).

V.-D. Nguyen is also with the University of Engineering and Technology, Vietnam National University, Hanoi, Vietnam.

H. Phan is with School of Electronic Engineering and Computer Science, Queen Mary University of London, United Kingdom (email: h.phan@qmul.ac.uk).

T. Marsault is with Département TEC/SPC, Direction générale de l'armement (DGA), Maitrise de l'information, France (email: thierry.marsault@intradef.gouv.fr) by accurate estimation but high computational complexity whereas the second group is represented by low computation complexity but inadequate accuracy. The Vogler method was developed based on a generalized residual series formulation [18] for electromagnetic waves over a sequence of smooth cylindrical obstacles. The obtained result of such derivation has the form of a multiple integral whose computation can be represented efficiently in terms of series representation. The Vogler method is the first of its kind allowing to predict a precise diffraction attenuation value up to ten knife-edges.

In terms of estimation accuracy, the Vogler method stands out from other methods [19], presenting the ultimate solution supported by both simulations and real-life measured experiment results [3], [19], [20]. For example, the Vogler method is also used as the ground truth in a comparative studies for fifty scenarios reported in [20]. Comparison results of multiple diffraction models for a digital broadcasting coverage prediction with different frequencies can be found in [3], [19].

However, the naive implementation of the Vogler algorithm results in high computational complexity, making it difficult to use in practice. To overcome this computational challenge, the recursive version was proposed by Vogler [21] to reduce the computational time. Unfortunately, this version came without a rigorous mathematical proof, but only the final result [21], [22], leading to difficult understanding and implementation. Thus, we aim to provide insight of the mathematical structure of this problem to gain a comprehensive interpretation. Our contributions are threefold. First, we analyze why the Vogler algorithm can be represented in a recursive manner. Second, we present a formal proof by induction. Finally, to gain intuition of the proof, we provide a case study of four knifeedges.

\section{THE VOGLER METHOD}



Fig. 1: Geometry of multiple knife edges.

In this section the background on the Vogler method will be presented. We consider the geometry of $N$ knife-edge 
diffraction in Fig. 1 where $h_{0}$ and $h_{N+1}$ denote the transmitter and receiver heights respectively, $\left\{h_{n}\right\}_{n=1}^{N}$ are the knife-edge heights to a reference surface, $\left\{\phi_{n}\right\}_{n=1}^{N}$ are diffraction angles, and $\left\{r_{n}\right\}_{n=1}^{N+1}$ are $N+1$ separation distances between knifeedges. Then the diffraction attenuation, $A_{N}$, for $N$ knife-edges is given by [22]

$$
\begin{aligned}
A_{N}= & \frac{1}{2^{N}} C_{N} \exp \left(\sigma_{N}\right)\left(\frac{2}{\sqrt{\pi}}\right)^{N} \underbrace{\int_{\beta_{1}}^{\infty} \cdots}_{N-\text { fold }} \int_{\beta_{N}}^{\infty} \\
& \exp (2 f) \exp \left(-\sum_{n=1}^{N} u_{n}^{2}\right) d u_{1} \cdots d u_{N}
\end{aligned}
$$

where

$$
\begin{aligned}
f & = \begin{cases}0 & \text { for } N=1 \\
\sum_{j=1}^{N-1} \gamma_{j} & \text { for } N \geq 2\end{cases} \\
\gamma_{j} & =\alpha_{j}\left(u_{j}-\beta_{j}\right)\left(u_{j+1}-\beta_{j+1}\right) \\
\alpha_{j} & =\left[\frac{r_{j} r_{j+1}}{\left(r_{j}+r_{j+1}\right)\left(r_{j+1}+r_{j+2}\right)}\right]^{1 / 2}, \text { where } 1 \leq j \leq N-1
\end{aligned}
$$$$
\beta_{j}=\phi_{j}\left[\frac{\mathrm{ik} r_{j} r_{j+1}}{2\left(r_{j}+r_{j+1}\right)}\right]^{1 / 2}, \text { where } 1 \leq j \leq N
$$$$
\sigma_{N}=\sum_{n=1}^{N} \beta_{n}^{2}
$$$$
C_{N}=\left\{\begin{array}{cc}
1 & \text { for } N=1 \\
{\left[\frac{\left(\sum_{n=1}^{N+1} r_{n}\right) \prod_{n=1}^{N} r_{n}}{\prod_{n=1}^{N}\left(r_{n}+r_{n+1}\right)}\right]^{1 / 2}} & \text { for } N \geq 2
\end{array}\right.
$$

with $\mathrm{i}=\sqrt{-1}$ being an imaginary number and the wave number $\mathrm{k}=2 \pi / \lambda$.

To calculate (1), the main idea in [22] is that instead of computing the $N$-fold integral, we convert such task into computing $N$ single integrals. To this end, Vogler proposed to express $\exp (2 f)$ in terms of power series as:

$$
\exp (2 f)=\sum_{m=0}^{\infty} \frac{(2 f)^{m}}{m !}
$$

Then by exploiting the fact that

$$
\frac{2}{\sqrt{\pi}} \int_{\beta}^{\infty}(u-\beta)^{m} \exp \left(-u^{2}\right) d u=m ! I(m, \beta)
$$

where $m$ ! refers to the factorial of $m$ and $I(m, \beta)$ defines the repeated integrals of the complementary error function [23], we reach a closed-form solution for $A_{N}$. The detail calculations are omitted here and can be found in [21]. The results are summarized in the following theorem

Theorem 1. For $N=1$, equation (1) is reduced to the standard form of a single knife-edge diffraction:

$$
A_{1}=\frac{1}{\sqrt{\pi}} \exp \left(\beta_{1}^{2}\right) \int_{\beta_{1}}^{\infty} \exp \left(-u^{2}\right) d u .
$$

For $N=2$, we reach a closed-form solution for $A_{2}$ as follows:

$$
A_{2}=\frac{1}{2^{2}} C_{2} \exp \left(\sigma_{2}\right) \sum_{m=0}^{\infty} I_{m}
$$

where

$$
I_{m}=2^{m} \alpha_{1}^{m} m ! I\left(m, \beta_{1}\right) I\left(m, \beta_{2}\right) .
$$

For $N>2$, a general solution for $A_{N}$ is given by:

$$
A_{N}=\frac{1}{2^{N}} C_{N} \exp \left(\sigma_{N}\right) \sum_{m=0}^{\infty} I_{m}
$$

where

$$
\begin{aligned}
& I_{m}= \\
& \quad 2^{m} \sum_{m_{1}=0}^{m} \ldots \sum_{m_{N-2}}^{m_{N-3}} \prod_{i=1}^{N} \frac{\left(m_{i-1}-m_{i+1}\right) !}{\left(m_{i}-m_{i+1}\right) !} \alpha_{i}^{m_{i-1}-m_{i}} I\left(n_{i}, \beta_{i}\right)
\end{aligned}
$$

with, by using notation $m_{0}=m$,

$$
n_{i}= \begin{cases}m_{0}-m_{1} & i=1 \\ m_{i-2}-m_{i} & 2 \leq i \leq N-1 . \\ m_{N-2}-m_{N-1} & i=N\end{cases}
$$

\section{ON THE RECURSIVE SOLUTION OF THE VOGLER METHOD}

Many existing studies in the literature highlight the high complexity of the Vogler method (see [3], [20], [24] and references therein). However, the technical report by Vogler [21] indeed includes a recursive version which provides a faster solution than the direct implementation. Unfortunately, there is a lack of a rigorous proof, making such version difficult for understanding and implementation. To overcome this problem, we provide here a mathematical proof of this recursive algorithm. We first show that the recursive structure of the Vogler algorithm stems from the structure of expansion of $f^{m}$. Then, we propose a general proof for the recursive algorithm (i.e., for $N>2$ ).

To show that the Vogler solution can provide a recursive structure, we first present the following lemma:

Lemma 1. Assume that $f$ has the following form as by (2) when $N>1$

$$
f=\sum_{i=1}^{N-1} \gamma_{i}
$$

for $N \geq 3$, we can represent $f^{m}$ as given by (17) and (18) at the top of the next page where

$$
\left(\begin{array}{l}
n \\
k
\end{array}\right)=\frac{n !}{(n-k) ! k !}
$$

denotes the binomial coefficient.

\section{Proof. See Appendix A.}

The non-recursive version of the Vogler algorithm is obtained by substituting (18) and (8) into (1) and then computing the integral to achieve (14). The main drawback of this version is that, considering two successive terms, $I_{m}$ and $I_{m+1}$, the computation of $I_{m+1}$ requires recalculating completely the sub-calculations in $I_{m}$, thus omitting the recursive structure of (17). By the recursive structure, we mean that $f^{m}$ can be represented in an abstract form as follows

$$
\begin{aligned}
& f^{m}\left(\gamma_{1}, \cdots, \gamma_{N-1}\right)=\psi\left(m, \gamma_{1}, \psi\left(m_{1}, \gamma_{2}, \psi(\cdots,\right.\right. \\
& \left.\left.\psi\left(m_{N-3}, \gamma_{N-2}, \gamma_{N-1}\right) \cdots\right)\right)
\end{aligned}
$$






or

$$
f^{m}=m ! \sum_{m_{1}=0}^{m} \sum_{m_{2}=0}^{m_{1}} \ldots \sum_{m_{N-2}=0}^{m_{N-3}} \prod_{i=1}^{N-1} \frac{\gamma_{i}^{m_{i-1}-m_{i}}}{\left(m_{i-1}-m_{i}\right) !}
$$

where

$$
\begin{aligned}
& 0 \leq m_{N-2} \leq \cdots \leq m_{1} \leq m, \\
& \psi(m, a, b)=\sum_{i=0}^{m}\left(\begin{array}{c}
m \\
i
\end{array}\right) b^{m-i} a^{i},
\end{aligned}
$$

Theorem 2. (Recursive computation of $I_{m}[22]$ ): Let

$$
\begin{aligned}
C(N & \left.-1, m_{N-2}, m_{N-3}\right) \\
& =\left(m_{N-3}\right) ! \alpha_{N-1}^{m_{N-2}} I\left(m_{N-3}, \beta_{N-1}\right) I\left(m_{N-2}, \beta_{N}\right) .
\end{aligned}
$$

Then, given the following notation

$$
\begin{aligned}
& i=m_{N-L}, j=m_{N-L-1}, k=m_{N-L-2} \\
& 2 \leq L \leq N-2, N \geq 4
\end{aligned}
$$

and the recursive relationship

$$
\begin{aligned}
C & (N-L, j, k) \\
& =\sum_{i=0}^{j} \frac{(k-i) !}{(j-i) !} \alpha_{N-L}^{j-i} I\left(k-i, \beta_{N-L}\right) C(N-L+1, i, j),
\end{aligned}
$$

the recursive computation of $I_{m}$ is given by

$$
I_{m}=2^{m} \sum_{m_{1}=0}^{m_{0}} \alpha_{1}^{m-m_{1}} I\left(m-m_{1}, \beta_{1}\right) C\left(2, m_{1}, m\right) .
$$

Proof. See Appendix B.

\section{CASe Study of Four KNIFE-Edges $(N=4)$}

To simplify the discussion and illustrate the main idea, we present here a case study of $N=4$. We note that by substituting (8) into (1), $A_{N}$ can be rewritten as

$$
A_{N}=\frac{1}{2^{N}} C_{N} \exp \left(\sigma_{N}\right) \sum_{m=0}^{\infty} I_{m}
$$

where

$$
\begin{aligned}
I_{m}= & \left(\frac{2^{m}}{m !}\right)\left(\frac{2}{\sqrt{\pi}}\right)^{N} \\
& \times \int_{\beta_{1}}^{\infty} \cdots \int_{\beta_{N}}^{\infty} f^{m} \exp \left(-\sum_{n=1}^{N} u_{n}^{2}\right) d u_{1} \cdots d u_{N},
\end{aligned}
$$

Since we aim to obtain a recursive formula for $I_{m}$, by substituting (17) into (27), $I_{m}$ for $N=4$ is given by

$$
\begin{aligned}
& I_{m}= \\
& \left(\frac{2^{m}}{m !}\right)\left(\frac{2}{\sqrt{\pi}}\right)^{4} \\
& \times \int_{\beta_{1}}^{\infty} \int_{\beta_{2}}^{\infty} \int_{\beta_{3}}^{\infty} \int_{\beta_{4}}^{\infty}\left(\sum_{m_{1}=0}^{m}\left(\begin{array}{c}
m \\
m_{1}
\end{array}\right) \gamma_{1}^{m-m_{1}} \sum_{m_{2}=0}^{m_{1}}\left(\begin{array}{c}
m_{1} \\
m_{2}
\end{array}\right) \gamma_{2}^{m_{1}-m_{2}} \gamma_{3} m_{2}\right) \\
& \quad \times \exp \left(-\sum_{n=1}^{4} u_{n}^{2}\right) d u_{1} \cdots d u_{4} .
\end{aligned}
$$

Using (3) and the definition of binomial coefficients (19), we can expand $I_{m}$ as follows

$$
\begin{aligned}
& I_{m}= \\
& \left(\frac{2^{m}}{m !}\right)\left(\frac{2}{\sqrt{\pi}}\right)^{4} \int_{\beta_{1}}^{\infty} \int_{\beta_{2}}^{\infty} \int_{\beta_{3}}^{\infty} \int_{\beta_{4}}^{\infty} \\
& \sum_{m_{1}=0}^{m} \frac{m !}{\left(m-m_{1}\right) ! m_{1} !} \alpha_{1}^{m-m_{1}}\left(u_{1}-\beta_{1}\right)^{m-m_{1}}\left(u_{2}-\beta_{2}\right)^{m-m_{1}} \\
& \times\left(\left(\sum_{m_{2}=0}^{m_{1}} \frac{m_{1} !}{\left(m_{1}-m_{2}\right) ! m_{2} !} \alpha_{2}^{m_{1}-m_{2}}\left(u_{2}-\beta_{2}\right)^{m_{1}-m_{2}}\right.\right. \\
& \left.\left.\quad \alpha_{3}^{m_{2}}\left(u_{3}-\beta_{3}\right)^{m_{1}}\left(u_{4}-\beta_{4}\right)^{m_{2}}\right)\right) \\
& \quad \times \exp \left(-\sum_{n=1}^{4} u_{n}^{2}\right) d u_{1} \cdots d u_{4} .
\end{aligned}
$$

Using (23), we can write

$$
C\left(3, m_{2}, m_{1}\right)=m_{1} ! \alpha_{3}^{m_{2}} I\left(m_{1}, \beta_{3}\right) I\left(m_{2}, \beta_{4}\right)
$$

which represents the first coefficient needed to compute the recursive form. Then, we can rewrite $I_{m}$ as

$$
\begin{aligned}
I_{m}= & \left(\frac{2^{m}}{m !}\right)\left(\frac{2}{\sqrt{\pi}}\right) \int_{\beta_{1}}^{\infty} \sum_{m_{1}=0}^{m} \frac{m !}{\left(m-m_{1}\right) !} \alpha_{1}^{m-m_{1}} \\
& \times\left(u_{1}-\beta_{1}\right)^{m-m_{1}} C\left(2, m_{1}, m\right) \exp \left(-u_{1}^{2}\right) d u_{1}
\end{aligned}
$$


where

$$
\begin{aligned}
& C\left(2, m_{1}, m\right)= \\
& \quad \sum_{m_{2}=0}^{m_{1}} \frac{\left(m-m_{2}\right) !}{\left(m_{1}-m_{2}\right) !} \alpha_{2}^{m_{1}-m_{2}} I\left(m-m_{2}, \beta_{2}\right) C\left(3, m_{2}, m_{1}\right) .
\end{aligned}
$$

Finally, by computing the integral of (31), we achieve

$$
\begin{aligned}
I_{m} & =\left(\frac{2^{m}}{m !}\right) \sum_{m_{1}=0}^{m} m ! \alpha_{1}^{m-m_{1}} \\
& \times\left[\frac{2}{\sqrt{\pi}} \int_{\beta_{1}}^{\infty} \frac{\left(u_{1}-\beta_{1}\right)^{m-m_{1}}}{\left(m-m_{1}\right) !} \exp \left(-u_{1}^{2}\right) d u_{1}\right] C\left(2, m_{1}, m\right) \\
& =2^{m} \sum_{m_{1}=0}^{m} \alpha_{1}^{m-m_{1}} I\left(m-m_{1}, \beta_{1}\right) C\left(2, m_{1}, m\right)
\end{aligned}
$$

which is the exact result of the Theorem 2 for $N=4$.

\section{CONClusion}

In this communication, we have introduced a rigorous proof for the recursive Vogler algorithm after analyzing its mathematical structure. A case study of four knife-edges is also presented in a 'closed form' to bring intuition of the key steps. Our study can provide a deep overview of the problem and clarify the algorithm derivation. Moreover, we could use the insight from the proof to develop an efficient recursive algorithm for the multiple bridged knife-edge diffraction problem [25]. The recursive Vogler algorithm can also be used to generate faster training dataset for function approximationbased methods [24], [26] proposed to bring a better trade-off in terms of the accuracy and the computational complexity.

\section{ACKNOWLEDGMENT}

We would like to thank the Direction générale de l'armement (DGA) and specially the Agence de l'Innovation de Défense (AID) for the financial support of our project "LINASAAF".

\section{APPENDIX}

\section{A. Proof of Lemma 1}

We obtain the first form (17) by applying the binomial theorem $N-2$ times to $f^{m}$

$$
\begin{aligned}
& \left(\sum_{i=1}^{N-1} \gamma_{i}\right)^{m}=\sum_{m_{1}=0}^{m}\left(\begin{array}{c}
m \\
m_{1}
\end{array}\right) \gamma_{1}^{m-m_{1}}\left(\sum_{i=2}^{N-1} \gamma_{i}\right)^{m_{1}} \\
& \left(\sum_{i=2}^{N-1} \gamma_{i}\right)^{m_{1}}=\sum_{m_{2}=0}^{m_{1}}\left(\begin{array}{l}
m_{1} \\
m_{2}
\end{array}\right) \gamma_{2}^{m_{1}-m_{2}}\left(\sum_{i=3}^{N-1} \gamma_{i}\right)^{m_{2}} \\
& \left(\gamma_{N-3}+\left(\gamma_{N-2}+\gamma_{N-1}\right)\right)^{m_{N-4}}= \\
& \sum_{m_{N-3}=0}^{m_{N-4}}\left(\begin{array}{l}
m_{N-4} \\
m_{N-3}
\end{array}\right) \gamma_{N-3}^{m_{N-4}-m_{N-3}}\left(\gamma_{N-2}+\gamma_{N-1}\right)^{m_{N-3}} \\
& \left(\gamma_{N-1}+\gamma_{N-2}\right)^{m_{N-3}}= \\
& \sum_{m_{N-2}=0}^{m_{N-3}}\left(\begin{array}{l}
m_{N-3} \\
m_{N-2}
\end{array}\right) \gamma_{N-2}^{m_{N-3}-m_{N-2}} \gamma_{N-1}^{m_{N-2}}
\end{aligned}
$$

To achieve the second form (18), we observe that, by rearranging the summation and the coefficients, (17) can be rewritten as

$$
f^{m}=\sum_{m_{1}=0}^{m} \sum_{m_{2}=0}^{m_{1}} \ldots \sum_{m_{N-2}=0}^{m_{N-3}} \prod_{i=1}^{N-1}\left(\begin{array}{l}
m_{i-1} \\
m_{i}
\end{array}\right) \gamma_{i}^{m_{i-1}-m_{i}}
$$

where we used the notation $m_{i}=0, i \geq N-1$. Note that,

$$
\prod_{i=1}^{N-1}\left(\begin{array}{l}
m_{i-1} \\
m_{i}
\end{array}\right)=\prod_{i=1}^{N-1} \frac{m_{i-1} !}{m_{i} !} \prod_{i=1}^{N-1} \frac{1}{\left(m_{i-1}-m_{i}\right) !}
$$

and, using again the notation $m_{0}=m$,

$$
\prod_{i=1}^{N-1} \frac{m_{i-1} !}{m_{i} !}=\frac{m_{0} !}{m_{1} !} \frac{m_{1} !}{m_{2} !} \cdots \frac{m_{N-2} !}{m_{N-1} !}=\frac{m_{0} !}{m_{N-1} !}=m !
$$

then, we obtain the desired result

$$
f^{m}=m ! \sum_{m_{1}=0}^{m} \sum_{m_{2}=0}^{m_{1}} \ldots \sum_{m_{N-2}=0}^{m_{N-3}} \prod_{i=1}^{N-1} \frac{\gamma_{i}^{m_{i-1}-m_{i}}}{\left(m_{i-1}-m_{i}\right) !} .
$$

This concludes the proof.

\section{B. Proof of Theory 2}

First, we denote the following set of integrals

$$
\begin{aligned}
\mathcal{I}_{1} & =\left(\frac{2}{\sqrt{\pi}}\right)^{2} \int_{\beta_{N-1}}^{\infty} \int_{\beta_{N}}^{\infty} \sum_{m_{N-2}=0}^{m_{N-3}}\left(\begin{array}{c}
m_{N-3} \\
m_{N-2}
\end{array}\right) \gamma_{N-2}^{m_{N-3}-m_{N-2}} \\
& \times \gamma_{N-1}^{m_{N-2}} \exp \left(-\left(u_{N-2}^{2}+u_{N-1}^{2}\right)\right) d u_{N-2} d u_{N-1}, \\
\mathcal{I}_{2} & =\left(\frac{2}{\sqrt{\pi}}\right)^{3} \int_{\beta_{N-2}}^{\infty} \int_{\beta_{N-1}}^{\infty} \int_{\beta_{N}}^{\infty} \sum_{m_{N-3}=0}^{m_{N-4}}\left(\begin{array}{l}
m_{N-4} \\
m_{N-3}
\end{array}\right) \gamma_{N-3}^{m_{N-4}-m_{N-3}} \\
& \times\left(\gamma_{N-2}+\gamma_{N-1}\right)^{m_{N-3}} \exp \left(-\sum_{i=N-2}^{N} u_{i}^{2}\right) d u_{N-2} \cdots d u_{N},
\end{aligned}
$$

$$
\begin{aligned}
& \mathcal{I}_{n}=\left(\frac{2}{\sqrt{\pi}}\right)^{n+1} \int_{\beta_{N-n}}^{\infty} \ldots \int_{\beta_{N}}^{\infty} \sum_{m_{N-n-1}=0}^{m_{N-n-2}}\left(\begin{array}{l}
m_{N-n-2} \\
m_{N-n-1}
\end{array}\right) \\
& \times \gamma_{N-n-1}^{m_{N-n-2}-m_{N-n-1}}\left(\sum_{i=N-n}^{N-1} \gamma_{i}\right)^{N-n-1} \\
& \quad \times \exp \left(-\sum_{i=N-n}^{N} u_{i}^{2}\right) d u_{N-n} \cdots d u_{N}, \\
& \vdots \\
& \mathcal{I}_{N-2}=\left(\frac{2}{\sqrt{\pi}}\right)^{N-1} \int_{\beta_{2}}^{\infty} \ldots \int_{\beta_{N}}^{\infty} \sum_{m_{1}=0}^{m}\left(\begin{array}{c}
m \\
m_{1}
\end{array}\right) \gamma_{1}^{m-m_{1}} \\
& \times\left(\sum_{i=2}^{N-1} \gamma_{i}\right)^{m_{1}} \exp \left(-\sum_{i=2}^{N} u_{i}^{2}\right) d u_{2} \cdots d u_{N} .
\end{aligned}
$$


We notice that, the following relationships hold:

$$
\begin{aligned}
\mathcal{I}_{n}= & \frac{2}{\sqrt{\pi}} \int_{\beta_{N-n}}^{\infty} \sum_{m_{N-n-1}=0}^{m_{N-n-2}}\left(\begin{array}{c}
m_{N-n-2} \\
m_{N-n-1}
\end{array}\right) \\
& \times \gamma_{m_{N-n-1}}^{m_{N-n-2}-m_{N-n-1}} \mathcal{I}_{n-1} \exp \left(u_{N-n}^{2}\right) d u_{N-n}
\end{aligned}
$$

for $2 \leq n \leq N-2$, and

$$
I_{m}=\left(\frac{2^{m}}{m !}\right) \frac{2}{\sqrt{\pi}} \int_{\beta_{1}}^{\infty} \mathcal{I}_{N-2} \exp \left(-u_{1}^{2}\right) d u_{1},
$$

stemming from definitions of $\mathcal{I}_{n}$ and $I_{m}$.

Our main idea is to find a general formula for $\mathcal{I}_{n}$. Then it is straightforward to obtain $I_{m}$ through (41). To this end, we will provide a general formula for $\mathcal{I}_{n}$ and prove it by induction. In particular, our proof can be divided into three steps:

- Step 1: We compute $\mathcal{I}_{1}$

- Step 2: We provide a general formula of $\mathcal{I}_{n}$ for $2 \leq n \leq$ $N-2$. We show that this formula is true by induction

- Step 3: We show that the results of Theorem 2 are consequences of Step 2, thus concluding the proof.

Now we provide the technical details.

Step 1: We compute $\mathcal{I}_{1}$

First, from (3) and (19), we note that

$$
\begin{aligned}
\gamma_{N-2}^{m_{N-3}} & -m_{N-2} \gamma_{N-1}^{m_{N-2}}= \\
\times & \alpha_{N-2}^{m_{N-3}-m_{N-2}}\left(u_{N-2}-\beta_{N-2}\right)^{m_{N-3}-m_{N-2}} \alpha_{N-1}^{m_{N-2}} \\
& \times\left(u_{N-1}-\beta_{N-1}\right)^{m_{N-3}}\left(u_{N}-\beta_{N}\right)^{m_{N-2}}
\end{aligned}
$$

Then by substituting (42) into (37), we obtain

$$
\begin{aligned}
\mathcal{I}_{1}= & \sum_{m_{N-2}=0}^{m_{N-3}} \frac{\left(m_{N-3}\right) !}{\left(m_{N-3}-m_{N-2}\right) !} \alpha_{N-2}^{m_{N-3}-m_{N-2}} \\
& \times\left(u_{N-2}-\beta_{N-2}\right)^{m_{N-3}-m_{N-2}} C\left(N-1, m_{N-2}, m_{N-3}\right)
\end{aligned}
$$

where

$$
\begin{aligned}
& C\left(N-1, m_{N-2}, m_{N-3}\right) \\
& \quad=\left(m_{N-3}\right) ! \alpha_{N-1}^{m_{N-2}} I\left(m_{N-3}, \beta_{N-1}\right) I\left(m_{N-2}, \beta_{N}\right) .
\end{aligned}
$$

Step 2: We provide formula of $\mathcal{I}_{n}$ for $2 \leq n \leq N-2$ and show that this formula is true by induction.

To this end, we introduce the following lemma

Lemma 2. For $2 \leq n \leq N-2, \mathcal{I}_{n}$ is given by

$$
\begin{aligned}
\mathcal{I}_{n}=\sum_{m_{N-n-1}=0}^{m_{N-n-2}} \frac{\left(m_{N-n-2}\right) !}{\left(m_{N-n-2}-m_{N-n-1}\right) !} \alpha_{N-n-1}^{m_{N-n-2}-m_{N-n-1}} \\
\quad \times\left(u_{N-n-1}-\beta_{N-n-1}\right)^{m_{N-n-2}-m_{N-n-1}} \\
\quad \times C\left(N-n, m_{N-n-1}, m_{N-n-2}\right)
\end{aligned}
$$

where

$C\left(N-n, m_{N-n-1}, m_{N-n-2}\right)=$

$\sum_{m_{N-n}=0}^{m_{N-n-1}} \frac{\left(m_{N-n-2}-m_{N-n}\right) !}{\left(m_{N-n-1}-m_{N-n}\right) !} \alpha_{N-n}^{m_{N-n-1}-m_{N-n}} \times$

$I\left(m_{N-n-2}-m_{N-n}, \beta_{N-n}\right) C\left(N-n+1, m_{N-n}, m_{N-n-1}\right)$.
Proof. We show that, this formula is true for $n=2$. Then we assume that (46) holds. Subsequently we prove that it is also true for $I_{n+1}$. Due to (40), we can rewrite (38) as:

$$
\begin{aligned}
\mathcal{I}_{2}= & \frac{2}{\sqrt{\pi}} \int_{\beta_{N-2}}^{\infty} \sum_{m_{N-3}=0}^{m_{N-4}}\left(\begin{array}{c}
m_{N-4} \\
m_{N-3}
\end{array}\right) \gamma_{N-3}^{m_{N-4}-m_{N-3}} \\
& \times \mathcal{I}_{1} \exp \left(-u_{N-2}^{2}\right) d u_{N-2} .
\end{aligned}
$$

By substituting (43) into (48) and compute the integral to the variable $u_{N-2}$, we have

$$
\begin{aligned}
\mathcal{I}_{2}= & \sum_{m_{N-3}=0}^{m_{N-4}} \frac{\left(m_{N-4}\right) !}{\left(m_{N-4}-m_{N-3}\right) !} \alpha_{N-3}^{m_{N-4}-m_{N-3}} \\
& \times\left(u_{N-3}-\beta_{N-3}\right)^{m_{N-4}-m_{N-3}} C\left(N-2, m_{N-3}, m_{N-4}\right)
\end{aligned}
$$

where

$$
\begin{aligned}
C(N- & \left.2, m_{N-3}, m_{N-4}\right) \\
= & \sum_{m_{N-2}=0}^{m_{N-3}} \frac{\left(m_{N-4}-m_{N-2}\right) !}{\left(m_{N-3}-m_{N-2}\right) !} \alpha_{N-2}^{m_{N-3}-m_{N-2}} \\
& \times I\left(m_{N-4}-m_{N-2}, \beta_{N-2}\right) C\left(N-1, m_{N-2}, m_{N-3}\right) .
\end{aligned}
$$

Now, assuming that (46) holds. We show that it is also true for $I_{n+1}$. In fact, because of (40), we have

$$
\begin{aligned}
& \mathcal{I}_{n+1}=\frac{2}{\sqrt{\pi}} \int_{\beta_{N-n-1}}^{\infty} \sum_{m_{N-n-2}=0}^{m_{N-n-3}}\left(\begin{array}{l}
m_{N-n-3} \\
m_{N-n-2}
\end{array}\right) \\
& \times \gamma_{m_{N-n-2}}^{m_{N-n-3}-m_{N-n-2}} \mathcal{I}_{n} \exp \left(u_{N-n-1}\right) d u_{N-n-1} .
\end{aligned}
$$

Substituting (46) into (51) and computing the integral to $u_{N-n-1}$ yield

$$
\begin{aligned}
& \mathcal{I}_{n+1}=\sum_{m_{N-n-2}=0}^{m_{N-n-3}} \frac{\left(m_{N-n-3}\right) !}{\left(m_{N-n-3}-m_{N-n-2}\right) !} \\
& \times \alpha_{N-n-2}^{m_{N-n-3}-m_{N-n-2}}\left(u_{N-n-2}-\beta_{N-n-2}\right)^{m_{N-n-3}-m_{N-n-2}} \\
& \times \sum_{m_{N-n-1}=0}^{m_{N-n-2}} \frac{\left(m_{N-n-3}-m_{N-n-1}\right) !}{\left(m_{N-n-2}-m_{N-n-1}\right) !} \alpha_{N-n-1}^{m_{N-n-2}-m_{N-n-1}} \\
& \times C\left(N-n, m_{N-n-1}, m_{N-n-2}\right) \\
& \times\left[\frac{2}{\sqrt{\pi}} \int_{\beta_{N-n-1}}^{\infty} \frac{\left(u_{N-n-1}-\beta_{N-n-1}\right)^{m_{N-n-3}-m_{N-n-1}}}{\left(m_{N-n-3}-m_{N-n-1}\right) !}\right. \\
& \left.\times \exp \left(u_{N-n-1}\right) d u_{N-n-1}\right] \\
& =\sum_{m_{N-n-2}=0}^{m_{N-n-3}} \frac{\left(m_{N-n-3}\right) !}{\left(m_{N-n-3}-m_{N-n-2}\right) !} \alpha_{N-n-2}^{m_{N-n-3}-m_{N-n-2}} \\
& \times\left(u_{N-n-2}-\beta_{N-n-2}\right)^{m_{N-n-3}-m_{N-n-2}} \\
& \times C\left(N-n-1, m_{N-n-2}, m_{N-n-3}\right)
\end{aligned}
$$


where

$$
\begin{aligned}
C(N- & \left.n-1, m_{N-n-2}, m_{N-n-3}\right) \\
= & \sum_{m_{N-n-1}=0}^{m_{N-n-2}} \frac{\left(m_{N-n-3}-m_{N-n-1}\right) !}{\left(m_{N-n-2}-m_{N-n-1}\right) !} \alpha_{N-n-1}^{m_{N-n-2}-m_{N-n-1}} \\
& \times I\left(m_{N-n-3}-m_{N-n-1}, \beta_{N-n-1}\right) \\
& \quad \times C\left(N-n, m_{N-n-1}, m_{N-n-2}\right)
\end{aligned}
$$

Thus, the formula (46) holds by induction, concluding the proof.

Step 3: We show that the results of Theorem 2 are consequences of Step 2, thus concluding the proof.

By using the relationship (41) and (47) with $n=N-2$, we obtain

$$
\begin{aligned}
I_{m}= & 2^{m} \sum_{m_{1}=0}^{m} \alpha_{1}^{m-m_{1}} C\left(2, m_{1}, m\right) \\
& \times\left[\frac{2}{\sqrt{\pi}} \int_{\beta_{1}}^{\infty} \frac{\left(u_{1}-\beta_{1}\right)^{m-m_{1}}}{\left(m-m_{1}\right) !} \exp \left(-u_{1}^{2}\right) d u\right] \\
= & 2^{m} \sum_{m_{1}=0}^{m} \alpha_{1}^{m-m_{1}} I\left(m-m_{1}, \beta_{1}\right) C\left(2, m_{1}, m\right) .
\end{aligned}
$$

Moreover, let

$$
\begin{aligned}
& m_{0}=m, \\
& i=m_{N-L}, j=m_{N-L-1}, k=m_{N-L-2} \\
& 2 \leq L \leq N-2, N \geq 4 .
\end{aligned}
$$

The following expression holds

$$
\begin{aligned}
& C(N-L, j, k) \\
& =\sum_{i=0}^{j} \frac{(k-i) !}{(j-i) !} \alpha_{N-L}^{j-i}(2 / \sqrt{\pi}) I\left(k-i, \beta_{N-L}\right) C(N-L+1, i, j) .
\end{aligned}
$$

This is a consequence from the proof of Lemma 2 (i.e., it holds by induction) on the recursive relationship of $C\left(N-1, m_{N-2}, m_{N-3}\right), C\left(N-2, m_{N-3}, m_{N-4}\right), \cdots$, $C\left(N-n, m_{N-n-1}, m_{N-n-2}\right)$ (see Equations (44), (50) and (47) respectively) and the identical form of terms in (17). We thus conclude the proof.

\section{REFERENCES}

[1] A. Goldsmith, Wireless communications. Cambridge university press, 2005.

[2] D. Erricolo and L. E. Uslenghi, "Two-dimensional simulator for propagation in urban environments," IEEE Transactions on Vehicular Technology, vol. 50, no. 4, pp. 1158-1168, 2001.

[3] S. R. Saunders and A. Aragón-Zavala, Antennas and propagation for wireless communication systems. John Wiley \& Sons, 2007.

[4] "Propagation by diffraction," International Telecommunication Union, Recommendation ITU-R P.526-15, 2019.

[5] T. S. Rappaport, G. R. MacCartney, S. Sun, H. Yan, and S. Deng, "Small-scale, local area, and transitional millimeter wave propagation for $5 \mathrm{~g}$ communications," IEEE Transactions on Antennas and Propagation, vol. 65, no. 12, pp. 6474-6490, Dec 2017.

[6] M. Boban, T. T. V. Vinhoza, M. Ferreira, J. Barros, and O. K. Tonguz, "Impact of vehicles as obstacles in vehicular ad hoc networks," IEEE Journal on Selected Areas in Communications, vol. 29, no. 1, pp. 15-28, January 2011.
[7] J. B. Andersen, "Transition zone diffraction by multiple edges," IEE Proceedings - Microwaves, Antennas and Propagation, vol. 141, no. 5, pp. 382-384, Oct 1994

[8] P. D. Holm, "UTD-diffraction coefficients for higher order wedge diffracted fields," IEEE Transactions on Antennas and Propagation, vol. 44, no. 6, pp. 879-888, June 1996.

[9] J. B. Andersen, "UTD multiple-edge transition zone diffraction," IEEE Transactions on Antennas and Propagation, vol. 45, no. 7, pp. 10931097, July 1997.

[10] M. Albani, F. Capolino, S. Maci, and R. Tiberio, "Diffraction at a thick screen including corrugations on the top face," IEEE Transactions on Antennas and Propagation, vol. 45, no. 2, pp. 277-283, 1997.

[11] C. Tzaras and S. R. Saunders, "An improved heuristic UTD solution for multiple-edge transition zone diffraction," IEEE Transactions on Antennas and Propagation, vol. 49, no. 12, pp. 1678-1682, Dec 2001.

[12] P. Valtr, P. Pechac, and M. Grabner, "Inclusion of higher order diffracted fields in the epstein-peterson method," IEEE Transactions on Antennas and Propagation, vol. 63, no. 7, pp. 3240-3244, July 2015.

[13] V. Daniele, G. Lombardi, and R. S. Zich, "The double PEC wedge problem: Diffraction and total far field," IEEE Transactions on Antennas and Propagation, vol. 66, no. 12, pp. 6482-6499, 2018.

[14] K. Bullington, "Radio propagation at frequencies above 30 megacycles," Proceedings of the IRE, vol. 35, no. 10, pp. 1122-1136, Oct 1947.

[15] J. Deygout, "Multiple knife-edge diffraction of microwaves," IEEE Transactions on Antennas and Propagation, vol. 14, no. 4, pp. 480489, July 1966.

[16] J. Causebrook and B. Davis, Tropospheric radio wave propagation over irregular terrain: The computation of field strength for UHF broadcasting. Research Department, Engineering Division, BBC, 1971.

[17] C. L. Giovaneli, "An analysis of simplified solutions for multiple knifeedge diffraction," IEEE Transactions on Antennas and Propagation, vol. 32, no. 3, pp. 297-301, March 1984.

[18] K. Furutsu, On the theory of radio wave propagation over inhomogeneous earth. National Bureau of Standards, Central Radio Propagation Laboratories, 1962

[19] C. Tzaras and S. R. Saunders, "Comparison of multiple-diffraction models for digital broadcasting coverage prediction," IEEE Transactions on Broadcasting, vol. 46, no. 3, pp. 221-226, Sep. 2000.

[20] N. DeMinco and P. McKenna, "A comparative analysis of multiple knifeedge diffraction methods," in Proc. ISART/ClimDiff, 2008, pp. 65-69.

[21] L. E. Vogler, "The attenuation of electromagnetic waves by multiple knife-edge diffraction," NASA STI/Recon Technical Report N, vol. 82, 1981.

[22] L. E. Vogler, "An attenuation function for multiple knife-edge diffraction," Radio Science, vol. 17, no. 06, pp. 1541-1546, Nov 1982.

[23] M. Abramowitz and I. A. Stegun, "Handbook of mathematical functions with formulas, graphs, and mathematical table," in US Department of Commerce. National Bureau of Standards Applied Mathematics series 55, 1965.

[24] J. Beyer, "An approximate approach to predict multiple screen diffraction in the case of grazing incidence," Radio Science, vol. 39, no. 4, pp. 1-6, Aug 2004.

[25] J. H. Whitteker, "A series solution for diffraction over terrain modeled as multiple bridged knife edges," Radio Science, vol. 28, no. 04, pp. 487-500, July 1993.

[26] V.-D. Nguyen, H. Phan, A. Mansour, and A. Coatanhay, "VoglerNet: Multiple knife-edge diffraction using deep neural network," in 2020 14th European Conference on Antennas and Propagation (EuCAP), 2020, pp. $1-5$ 